\title{
Two staging systems for gastrointestinal stromal tumors in the stomach: which is better?
}

\author{
Chul Hong Park', Gwang Ha Kim, Bong Eun Lee', Geun Am Song ${ }^{1}$, Do Youn Park², Kyung Un Choi²,
} Dae Hwan $\mathrm{Kim}^{3}$ and Tae Yong Jeon ${ }^{3}$

\begin{abstract}
Background: The prognosis of a gastrointestinal stromal tumor (GIST) is influenced by its anatomic site; however, few studies on the prognosis of gastric GISTs have been reported. The aims of this study were to evaluate long-term prognoses of patients who underwent surgical resection for gastric GISTs and to compare the clinical efficacy of two staging systems: the National Institutes of Health $(\mathrm{NIH})$ consensus criteria and the 7th Union for International Cancer Control/American Joint Committee on Cancer (UICC/ AJCC) tumor-node-metastasis (TNM) staging system.
\end{abstract}

Methods: We conducted a retrospective observational study of 145 patients who underwent surgical resection for gastric GISTs between February 2001 and June 2012 at Pusan National University Hospital (Busan, Korea). Recurrence and 5-year recurrence-free survival (RFS) rates were analyzed.

Results: During a median follow-up period of 44 months (range, 6-144 months), 11 recurrent lesions were detected in 9 patients (6.4\%). On multivariate analysis, tumor size $(>5 \mathrm{~cm})$, mitotic count ( $>5 / 50$ high-power fields), and epithelioid and mixed pathological type were significantly associated with recurrence. The overall 5-year RFS rate was $93.4 \%$. Although no statistically significant differences were detected ( $C$-statistic difference $P=0.886$ ), all metrics showed lower values for the UICC/AJCC TNM staging system than for the NIH consensus criteria, suggesting that the UICC/AJCC TNM staging system may be a better model.

Conclusions: The 5-year RFS rate in patients who underwent curative resection for gastric GISTs was excellent. The UICC/ AJCC TNM staging system may be more useful than the NIH consensus criteria for risk categorization of patients with gastric GISTs.

Keywords: Stomach, Gastrointestinal stromal tumors, Staging, Prognosis, Recurrence

\section{Background}

Gastrointestinal stromal tumors (GISTs) are the most common mesenchymal tumors of the gastrointestinal tract. GISTs originate from intestinal pacemaker cells, known as interstitial cells of Cajal [1]. The pathogenesis of GISTs is related to oncogenic mutations in either the KIT or PDGFR $\boldsymbol{\alpha}$ genes [2, 3]. GISTs can occur anywhere along the gastrointestinal tract. The most common site is

\footnotetext{
* Correspondence: doc0224@pusan.ac.kr

${ }^{1}$ Department of Internal Medicine, Pusan National University School of Medicine, and Biomedical Research Institute Pusan National University Hospital, 179, Gudeok-ro, Seo-Gu, Busan 49241, South Korea Full list of author information is available at the end of the article
}

the stomach (60\%), followed by the jejunum and ileum (30\%), duodenum (4-5\%), rectum (4\%), colon and appendix $(1-2 \%)$, and esophagus $(<1 \%)$ [4]. These tumors rarely occur outside the gastrointestinal tract at sites such as the omentum, mesentery, or retroperitoneum [4].

GISTs are characterized by a diverse spectrum of morphological and clinical features ranging from benign to malignant. Approximately $10-30 \%$ of GISTs are clinically malignant, but all GISTs are considered to have some degree of malignant potential [5]. The primary treatment for localized GISTs is complete surgical resection with microscopic negative margins. Patients with unresectable, metastatic, or recurrent GISTs can be treated with 
imatinib mesylate. Moreover, adjuvant treatment with imatinib mesylate after surgical resection is proven to improve recurrence-free survival (RFS) of patients with GISTs [6-8].

To assess risk in the clinical course of GISTs, a consensus conference held at the National Institutes of Health $(\mathrm{NIH})$ in 2001 provided the first practical scheme for risk categorization based on tumor size and mitotic index (per 50 high-power fields [HPF]) [2]. Since that time, the NIH consensus criteria have been most commonly used in clinical studies. However, these criteria do not consider the primary location of GISTs in predicting risk. Therefore, based on the different clinical outcomes of GISTs according to primary tumor site, the 7th Union for International Cancer Control/American Joint Committee on Cancer (UICC/AJCC) tumor-node-metastasis (TNM) staging system was proposed in 2010 [9].

Generally, the prognoses of GISTs are influenced by their anatomic sites. It has been reported that patients with gastric GISTs have better prognoses than those with non-gastric GISTs [4, 10-12]. While many studies have reported prognoses of all GISTs irrespective of their primary anatomic sites, studies specific to gastric GISTs are few in number. A recent retrospective multicenter study of 1057 gastric GISTs suggested that Eastern patients with gastric GISTs had more favorable outcomes than Western patients [13]. Therefore, we aimed to investigate the longterm prognoses of patients with gastric GISTs who underwent curative surgical resection and to compare the clinical efficacy of the 7th UICC/AJCC TNM staging system with that of the NIH consensus criteria.

\section{Methods}

\section{Patients}

The study cohort consisted of 155 patients who underwent surgery for gastric GISTs at Pusan National University Hospital (Busan, Korea) between February 2001 and June 2012. The inclusion criterion was patients who underwent curative surgical resection for primary gastric GISTs. The exclusion criteria were distant metastasis at the time of surgery, preoperative chemotherapy with imatinib and the presence of another concomitant malignancy. Ten patients were excluded because they were incidentally discovered to have gastric GISTs during gastrectomy for gastric cancer. Finally, 145 patients with gastric GISTs were included in this study. Written informed consent was obtained from all patients, and the study protocol was reviewed and approved by the Institutional Review Board of Pusan National University Hospital (E - 2015216).

\section{Histopathology}

All resected specimens were examined by two expert pathologists (DY Park and KU Choi). Paraffin-embedded resected specimens were sectioned and stained with hematoxylin and eosin. A diagnosis of GIST was confirmed by immunohistochemical staining for c-Kit (CD117) or DOG-1 and CD34. Histologic features such as tumor location, tumor size, mitotic count, or histologic subtype (spindle, epithelioid, or mixed) were also evaluated (Fig. 1). A tumor's size was defined as its largest diameter. The mitotic count of a tumor was evaluated as the number of mitosis per $50 \mathrm{HPF}$. In discordant cases, the histopathologic findings were adjudicated by consensus between the two pathologists.

Tumor staging was performed according to the $\mathrm{NIH}$ consensus criteria and the 7th UICC/AJCC TNM staging system. Under the NIH consensus criteria, tumors were categorized as very low risk, low risk, intermediate risk, and high risk based on mitotic activity and tumor size, as shown in Table 1 [2]. Under the 7th UICC/AJCC TNM staging system, tumors were categorized into 4 T-categories based solely on tumor size; the T-category was then associated with the mitotic rate. The presence of either nodal or distant metastasis indicates stage IV disease [9].

\section{Follow-up}

Postoperative follow-up information was obtained at the regular outpatient visits. Each patient's medical history was obtained, and all underwent a physical examination, esophagogastroduodenoscopy, and abdominal pelvic computed tomography 6 months after surgery and annually thereafter. All patients underwent the same followup protocol regardless of risk stratification. A diagnosis of recurrence was based on clinical, radiologic or endoscopic findings.

\section{Statistical analyses}

Categorical variables are expressed as a percentage and continuous variables are expressed by median and range. Tumor size was divided into two groups $(\leq 5 \mathrm{~cm}$ and $>5 \mathrm{~cm}$ ), and mitotic count was grouped into two categories ( $\leq 5 / 50 \mathrm{HPF}$ and $>5 / 50 \mathrm{HPF})$. Univariate and multivariate survival analyses included demographic and clinical variables such as sex, age, tumor size, mitotic count, histologic subtype, or operative method. Hazard ratios (HRs) and $95 \%$ confidence intervals (CIs) were calculated using the Cox proportional hazard model. RFS was defined as the interval between the date of surgery and either the date of the last follow-up or the date of recurrence, and was calculated by using the Kaplan-Meier method. Model fit statistics such as the $-2 \log$ likelihood ratio, Akaike's Information Criterion (AIC), or Schwarz Bayesian Criterion $(\mathrm{SBC})$ were used to compare the two different risk stratification methods. The better model was indicated by a lower value of each statistic. A $P$ value $<0.05$ was regarded statistically significant. All data were analyzed by the SAS software, version 9.3 (SAS Institute, Cary, NC, USA) and R software, version 3.2.2 (R Foundation for Statistical Computing, Vienna, Austria). 


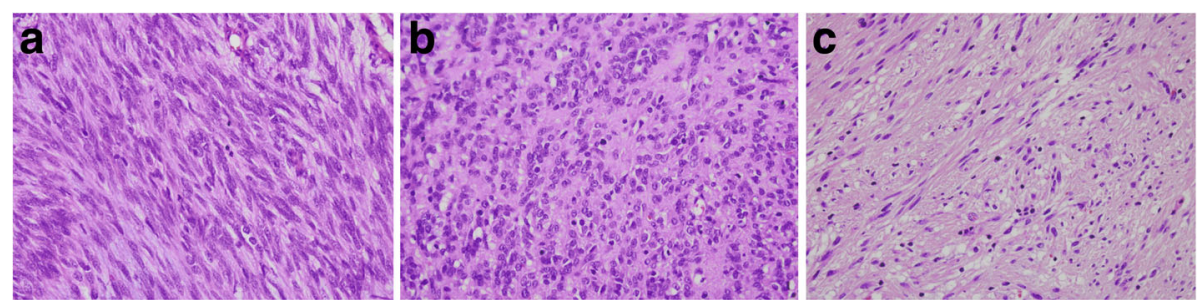

Fig. 1 Histologic subtypes of GISTs. a Spindle cell type. The tumor is composed of fusiform spindle cells with a fascicular growth pattern. $\mathbf{b}$ Epithelioid type. The tumor is composed of large round or polygonal cells with abundant, often eosinophilic or clear cytoplasm. c Mixed type. The tumor is composed of mixture of both types (hematoxylin and eosin staining, original magnification x400).

\section{Results}

\section{Clinicopathologic characteristics of patients with gastric GISTs}

Clinicopathologic characteristics of the 145 patients who underwent surgical resection for gastric GISTs are summarized in Table 2. The patients included 64 men and 81 women with a median age of 59 years (range, 479 years). Eighty-one tumors were located in the upper third of the stomach, 52 in the middle third, and 12 in the lower third. GISTs were resected via laparoscopic surgery in 86 patients and open surgery in 59 patients. Complete resection was achieved in 144 patients (99.3\%); only one case had a ruptured GIST at the time of diagnosis. The median tumor size was $3.1 \mathrm{~cm}$ (range, $0.7-26 \mathrm{~cm})$. Most tumors $(120 / 145 ; 82.8 \%)$ were $\leq 5 \mathrm{~cm}$ in maximum diameter. The mitotic figure count was $\leq 5 /$ $50 \mathrm{HPF}$ in 98 tumors, $>5 / 50 \mathrm{HPF}$ and $\leq 10 / 50 \mathrm{HPF}$ in 25 tumors, and $>10 / 50 \mathrm{HPF}$ in 22 tumors. The predominant histologic subtype was spindle cell type (130/145, 89.7\%). Six patients (4.1\%) were administered imatinib mesylate as adjuvant treatment.

According to the $\mathrm{NIH}$ consensus criteria, 25 patients $(17.2 \%)$ were in the very low risk group, 64 (44.1\%) in the low risk group, 23 (15.9\%) in the intermediate risk group, and $33(22.8 \%)$ in the high risk group. According to the 7 th UICC/AJCC TNM staging system, 89 patients

Table 1 The NIH consensus criteria and the 7th UICC/AJCC TNM staging system for gastric GISTs

\begin{tabular}{llll}
\hline $\begin{array}{l}\text { Tumor size } \\
(\mathrm{cm})\end{array}$ & $\begin{array}{l}\text { Mitotic count } \\
(/ 50 \mathrm{HPF})\end{array}$ & NIH criteria & UICC/AJCC TNM stage \\
\hline$\leq 2$ & $\leq 5$ & Very low & IA \\
$>2, \leq 5$ & $\leq 5$ & Low & IA \\
$>5, \leq 10$ & $\leq 5$ & Intermediate & IB \\
$>10$ & $\leq 5$ & Intermediate & $\|$ \\
$\leq 2$ & $>5$ & Intermediate & $\|$ \\
$>2, \leq 5$ & $>5$ & Intermediate & $\|$ \\
$>5, \leq 10$ & $>5$ & High & IIIA \\
$>10$ & $>5$ & High & IIIB \\
\hline
\end{tabular}

HPF high-power fields

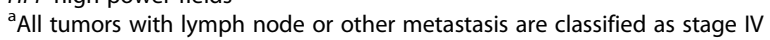

(61.4\%) were stage IA, 7 (4.8\%) were IB, 34 (23.4\%) were II, 10 (6.9\%) were IIIA, and 5 (3.4\%) were IIIB.

\section{Recurrence}

During the median follow-up period of 44 months (range, 6-144 months), 9 patients (6.2\%) experienced recurrence; of these patients, recurrence was histopathologically confirmed in 3 . The most common site was the

Table 2 Baseline clinicopathologic characteristics of 145 patients who underwent surgical resection for gastric GISTS

\begin{tabular}{ll}
\hline & No. of patients (\%) \\
\hline Median age, years (range) & $59(4-79)$ \\
Sex, $n$ (\%) & \\
Male & $64(44.1)$ \\
Female & $81(55.9)$ \\
Tumor location, $n$ (\%) & \\
$\quad$ Upper third & $81(55.9)$ \\
Middle third & $52(35.9)$ \\
Lower third & $12(8.3)$ \\
Median tumor size, cm (range) & $3.1(0.7-26)$ \\
Tumor size (cm), $n$ (\%) & \\
$\leq 2$ & $29(20.0)$ \\
$>2, \leq 5$ & $91(62.8)$ \\
$>5, \leq 10$ & $17(11.7)$ \\
$>10$ & $8(5.5)$ \\
Mitotic count (per 50 high-power fields), $n(\%)$ & \\
$\leq 5$ & $98(67.6)$ \\
$>5, \leq 10$ & $25(17.2)$ \\
$>10$ & $22(15.2)$ \\
Histologic subtype, $n$ (\%) & \\
Spindle type & $13(9.0)$ \\
Epithelioid type & \\
Operative method, $n$ (\%) & \\
Laparoscopic surgery & \\
\hline & \\
&
\end{tabular}


liver (45.4\%), followed by the peritoneal cavity (27.3\%), site of surgery (18.2\%), and spleen (9.1\%) (Table 3). According to the NIH consensus criteria, all nine cases were in the intermediate or high risk groups; recurrence was observed in 3 (13.0\%) of 23 intermediate risk cases and $6(18.2 \%)$ of 33 high risk cases. According to the 7 th UICC/AJCC TNM staging system, tumor recurrence occurred in one stage I case, three stage II cases, four stage IIIA cases, and one stage IIIB case. The recurrence rates at each stage were $1.0 \%$ in stage I, $8.8 \%$ in stage II, $40.0 \%$ in stage IIIA, and $20.0 \%$ in stage IIIB. All nine patients were treated with imatinib mesylate after recurrent disease was confirmed.

Factors associated with recurrence in patients with gastric GISTs

Univariate analysis revealed that tumor size $(>5 \mathrm{~cm})$, mitotic count ( $>5 / 50 \mathrm{HPF}$ ), and histologic subtype (epithelioid and mixed type) were associated with recurrence (Table 4). When all six variables were included in the Cox proportional hazard regression model, tumor size $>5 \mathrm{~cm}$ (HR, 10.75; 95\% CI, 2.12-54.60; $P=0.004)$, mitotic count $>5 / 50 \mathrm{HPF}$ (HR, 10.55; 95\% CI, 1.25-88.85; $P=0.030)$, and epithelioid and mixed type (HR, 5.73; 95\% CI, $1.29-25.53 ; P=0.022$ ) were significantly associated with recurrence via multivariate analyses (Table 4).

Table 3 Long-term outcomes of 145 patients who underwent surgical resection for gastric GISTS

\begin{tabular}{ll}
\hline & No. of patients (\%) \\
\hline Median follow-up duration, month (range) & $44(6-144)$ \\
Recurrence, $n$ (\%) & \\
Yes & $9(6.2)$ \\
No & $136(93.8)$ \\
Recurrence site ${ }^{a}, n$ (\%) & \\
Liver & $5(45.5)$ \\
Peritoneum & $3(27.3)$ \\
Spleen & $2(18.2)$ \\
Operation site & $1(9.1)$ \\
Recurrence according to NIH consensus criteria & \\
Very low & $0 / 25(0)$ \\
Low & $0 / 65(0)$ \\
Intermediate & $3 / 23(13.0)$ \\
High & $6 / 33(18.2)$ \\
Recurrence according to 7th UICC/AJCC TNM staging system \\
I & $1 / 96(1.0)$ \\
II & $3 / 34(8.8)$ \\
IIIA & $4 / 10(40)$ \\
IIIB & $1 / 5(20)$ \\
\hline
\end{tabular}

${ }^{a} 11$ recurrent lesions were observed in 9 patients 5-year recurrence-free survival rates in patients with gastric GISTs

The overall 5-year RFS rate was 93.4\%. According to the $\mathrm{NIH}$ consensus criteria, 5-year RFS rates were $100.0 \%$ in the very low and low risk groups, $88.9 \%$ in the intermediate risk group, and $80.1 \%$ in the high risk group (Fig. 2). According to the 7th UICC/AJCC TNM staging system, 5 -year RFS rates were $98.7 \%$ in stage I, $93.1 \%$ in stage II, $74.1 \%$ in stage IIIA and $60 \%$ in stage IIIB (Fig. 3 ).

\section{Comparison of 5-year recurrence-free survival rates according to the NIH consensus criteria and the 7th UICC/AJCC TNM staging system}

The 5-year RFS rates were at least 93\% in the stage I and II group by the 7th UICC/AJCC TNM staging system and were above $89 \%$ in the very low, low, and intermediate risk groups by the NIH consensus criteria. Stage III or high risk groups showed lower RFS rates. Two risk stratification classifications were redistributed to the high risk and low risk groups. The high risk group included high risk according to the NIH consensus criteria and stage IIIA and IIIB according to the 7th UICC/AJCC TNM staging system; the low risk group included very low, low, and intermediate risk according to the NIH classification and stage I and II according to the 7th UICC/AJCC TNM staging system. Accordingly, model fit statistics including the $-2 \log$ likelihood ratio, AIC and SBC were used to compare the two risk stratification models. Although no statistically significant difference was detected (C-statistic difference $P=0.886$ ), all three metrics showed lower values for the 7th UICC/AJCC TNM staging system than for the $\mathrm{NIH}$ consensus criteria, indicating that the 7th UICC/AJCC TNM staging system may be a better model (Table 5 ).

\section{Discussion}

In the present study, the 5-year RFS rate in patients who underwent surgery for gastric GISTs was excellent. This was influenced significantly by tumor size, mitotic count, and histologic subtype. No differences were detected in distributions of risk groups predicting the 5-year RFS rates between the NIH consensus criteria and 7th UICC/AJCC TNM staging system. To our knowledge, this is the first study to compare these two staging systems in patients with gastric GISTs.

Although many previous studies have reported the clinical outcomes of GISTs, few studies have focused on gastric GISTs alone. Miettinen et al. first published a largescale, retrospective study of 1765 patients with gastric GISTs, reporting on their clinicopathologic features and improved prognoses [14]. Thereafter, several studies have shown that the tumor site should be included for risk stratification of GISTs in addition to the tumor size and mitotic count of the primary tumor $[4,9]$. In particular, the prognosis for gastric GISTs is significantly better than 
Table 4 Factors associated with recurrence in patients who underwent surgical resection for gastric GISTS

\begin{tabular}{|c|c|c|c|c|c|c|}
\hline \multirow[t]{2}{*}{ Variables } & \multicolumn{3}{|c|}{ Univariate Analysis } & \multicolumn{3}{|c|}{ Multivariate Analysis } \\
\hline & $\mathrm{HR}$ & $95 \% \mathrm{Cl}$ & $P$-value & $\mathrm{HR}$ & $95 \% \mathrm{Cl}$ & $P$-value \\
\hline \multicolumn{7}{|l|}{ Sex } \\
\hline Male & 1 & & & & & \\
\hline Female & 0.58 & $0.16-2.17$ & 0.420 & & & \\
\hline \multicolumn{7}{|l|}{ Age (years) } \\
\hline$\leq 60$ & 1 & & & & & \\
\hline$>60$ & 2.64 & $0.66-10.54$ & 0.171 & & & \\
\hline \multicolumn{7}{|l|}{ Tumor size $(\mathrm{cm})$} \\
\hline$\leq 5$ & 1 & & & 1 & & \\
\hline$>5$ & 16.58 & $3.44-79-83$ & $<0.001$ & 10.75 & $2.12-54.60$ & 0.004 \\
\hline \multicolumn{7}{|c|}{$\begin{array}{l}\text { Mitotic count } \\
\text { (per } 50 \text { high-power fields) }\end{array}$} \\
\hline$\leq 5$ & 1 & & & 1 & & \\
\hline$>5$ & 16.72 & $2.09-133.95$ & 0.008 & 10.55 & $1.25-88.85$ & 0.030 \\
\hline \multicolumn{7}{|l|}{ Histologic subtype } \\
\hline Spindle type & 1 & & & 1 & & \\
\hline $\begin{array}{l}\text { Epithelioid and } \\
\text { mixed type }\end{array}$ & 4.48 & $1.12-18.00$ & 0.034 & 5.73 & $1.29-25.53$ & 0.022 \\
\hline \multicolumn{7}{|l|}{ Operative method } \\
\hline $\begin{array}{l}\text { Laparoscopic } \\
\text { surgery }\end{array}$ & 1 & & & & & \\
\hline Open surgery & 4.76 & $0.99-22.93$ & 0.052 & & & \\
\hline
\end{tabular}

HR hazard ratio, $\mathrm{Cl}$ confident interval for non-gastric GISTs [4, 9, 12]. The 5-year disease free survival rate of patients with gastric GISTs is $67.5 \%$, which is higher than that of patients with non-gastric GISTs (36.5\%) [12].

In the present study, the recurrence rate of gastric GISTs after surgery was $6.2 \%$, which is lower than the recurrence rates of 17 to $24 \%$ reported in previous Western studies $[15,16]$. On the other hand, the recurrence rate in our study is similar to the recurrence rate of 2.7 to $8.9 \%$ in recent studies including Korean and Japanese patients with gastric GISTs [13, 17]. On the basis of these results, Eastern patients with gastric GISTs may have more favorable outcomes than Western patients. The common sites of recurrence in the present study were the liver and peritoneal cavity, similar to those observed in previous studies [13, 15-17].

In the present study, multivariate analyses revealed that tumor size, mitotic count, and histologic subtype were associated with recurrence after curative resection for gastric GISTs. Most studies have shown that the most important prognostic factors for GISTs are tumor size and mitotic count $[14,18,19]$. In the case of histologic subtype, some studies have reported that recurrence is not associated with histologic subtype [20,21]. On the other hand, another study showed that patients with spindle cell type GIST show significantly higher 5year RFS than those with epithelioid or mixed type, which is similar to our results [22].

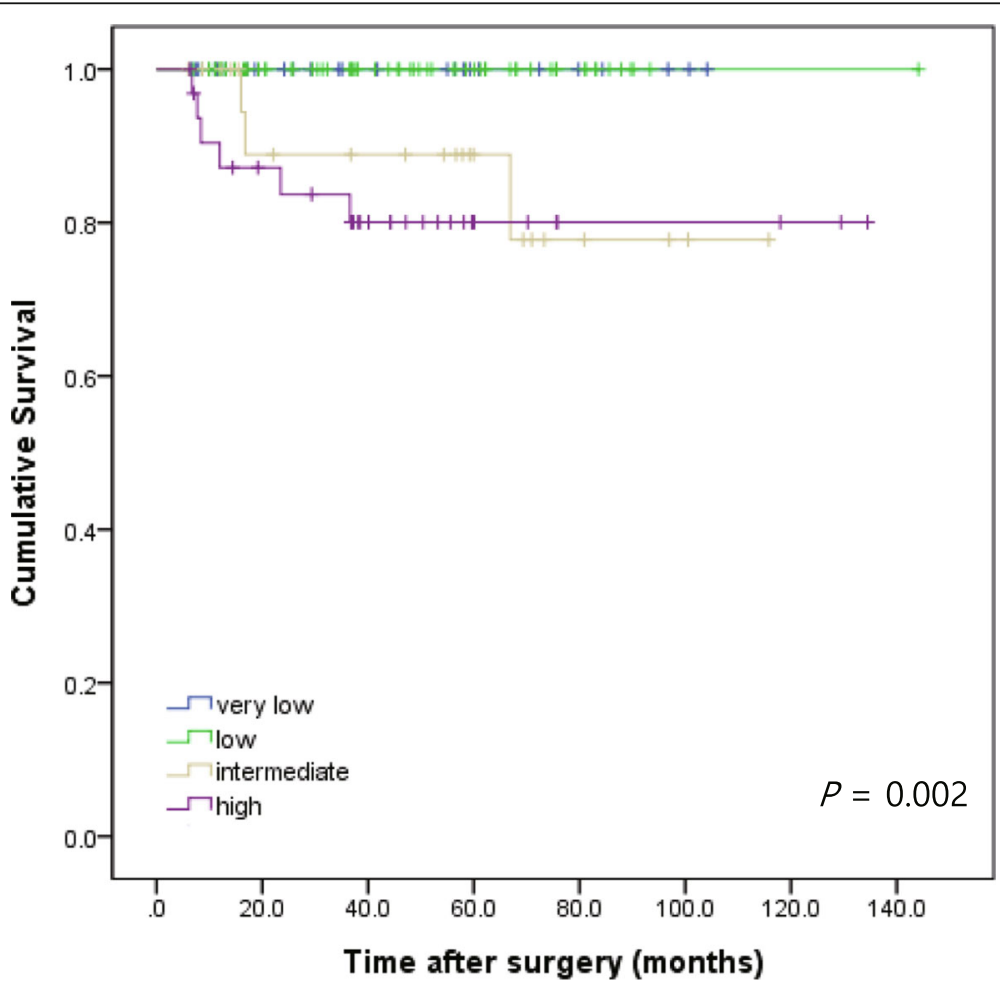

Fig. 2 Recurrence-free survival rate of patients undergoing surgical resection for gastric GISTs according to the NIH consensus criteria 


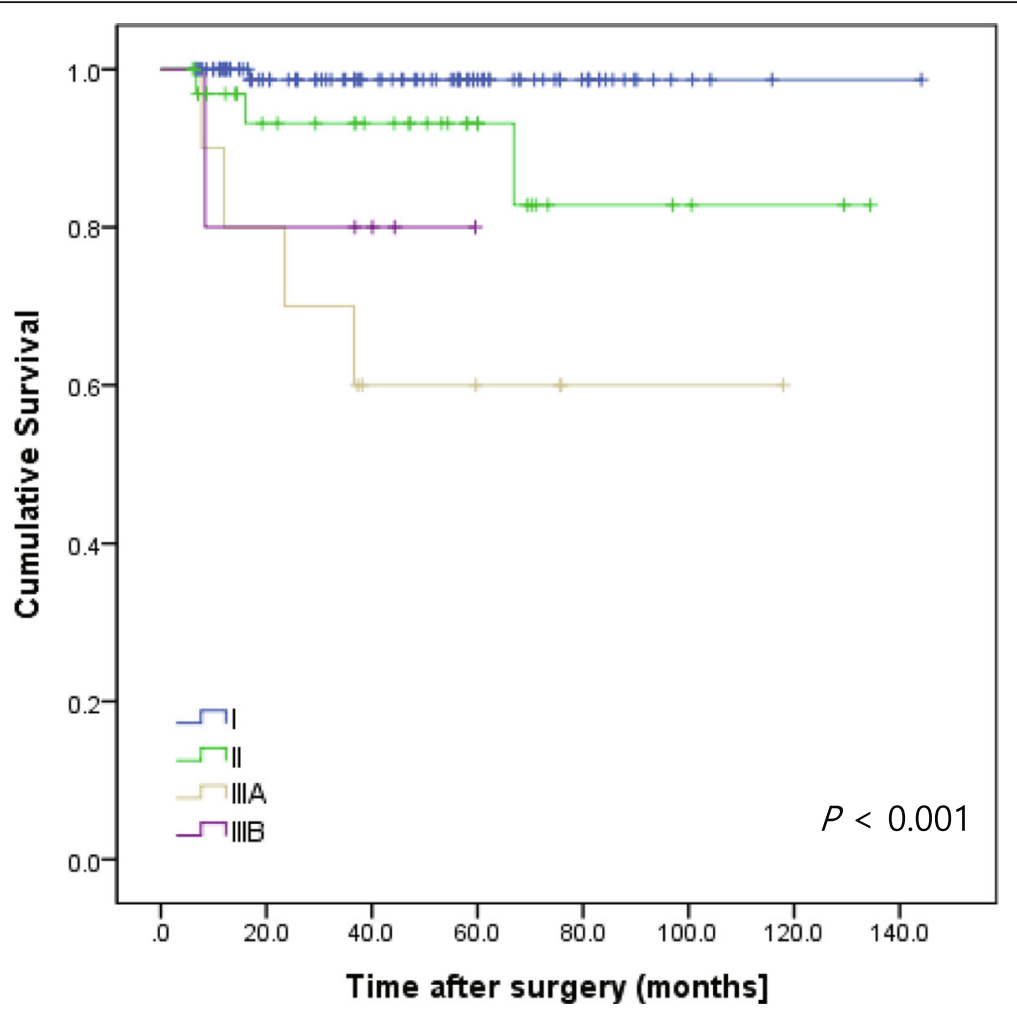

Fig. 3 Recurrence-free survival rate of patients undergoing surgical resection for gastric GISTs according to the $7^{\text {th }}$ UICC/AJCC TNM staging system

The median age at the time of diagnosis was 59 years, and the median tumor size was $3.1 \mathrm{~cm}$ in our study. Compared with Western studies [14, 23, 24], patients with gastric GISTs in our study were younger and had smaller tumors. Eastern studies conducted in Korea and Japan showed similar results to our study [13, 17]. These observed differences between Western and Eastern patients might be attributable to nationwide gastric cancer screening programs for people aged 40 years and older every 2 years provided by the Korea and Japan governments [25, 26]. Therefore, Eastern patients with gastric GISTs may have more favorable outcomes than Western patients.

In the present study, 5-year RFS rates according to the $\mathrm{NIH}$ consensus criteria were $100 \%$ in the very low risk, $100 \%$ in the low risk group, $88.9 \%$ in the intermediate risk group, and $80.1 \%$ in the high risk group; those according to the 7th UICC/AJCC TNM staging system were $98.7 \%$ in stage I, $93.1 \%$ in stage II, $74.1 \%$ in stage IIIA, and $60 \%$ in stage IIIB. These results are similar to those reported in previous Eastern studies [13, 17], and superior to those reported in Western studies [12, 14, 23, 24].

We evaluated the applicability of the 7th UICC/AJCC TNM staging system as compared to the NIH consensus criteria. The goodness of fit of the 7th UICC/AJCC TNM staging system was higher than that of the NIH consensus criteria, although no significant difference was observed. In a previous study comparing the 7th UICC/AJCC TNM staging system and the NIH consensus criteria, although the authors did not use a statistical method to compare both systems as in our study, they suggested that the 7th UICC/AJCC TNM staging system could reflect the 5-year RFS better than the NIH consensus criteria [13]. Therefore, although no significant difference was observed between these two systems, the 7th UICC/AJCC TNM staging system may be more useful in risk categorization

Table 5 Comparison of the NIH consensus criteria and the 7th UICC/AJCC TNM staging system in predicting recurrence free survival in patients who underwent surgical resection for gastric GISTS

\begin{tabular}{llllll}
\hline & Unadjusted & Model Fit Statistics & & & \\
\cline { 2 - 6 } & HR $(95 \%$ Cl) & -2 Log Likelihood & AIC & SBC & C-statistic (95\% CI) \\
\hline NIH (high/very low-intermediate) & $7.041(1.755-28.248)$ & 75.489 & 77.489 & 77.687 & $0.758(0.615-0.901)$ \\
AJCC (III/-II) & $10.579(2.835-39.482)$ & 72.618 & 74.618 & 74.816 & $0.745(0.639-0.851)$ \\
\hline
\end{tabular}

AIC Akaike's Information Criterion, SBC Schwarz Bayesian Criterion

${ }^{a}$ Overall adequacy of risk prediction procedures with censored survival data 
in patients with gastric GISTs than the NIH consensus criteria.

Our study has some limitations. First, the present study was a single-center retrospective study and, as a result, there might have been potential selection biases. Treatment options were selected on a case-by-case basis according to clinical judgment and patient factors. Second, our study had a relatively small number of patients and a short follow-up period. Further large-scale studies with longer follow-up periods are needed to clarify the clinical efficacy of the 7th UICC/AJCC TNM staging system. Third, because we focused only on clinical outcomes in patients who underwent curative resection for gastric GISTs, we did not include patients who had distant metastases at the time of surgery or who underwent preoperative or postoperative chemotherapy. Lastly, we included patients with only gastric GISTs; our results ought to be validated in studies that include GISTs in all sites. The clinical efficacy of the 7th UICC/AJCC TNM staging system and that of the NIH consensus criteria can then be compared according to the tumor's primary anatomic site.

\section{Conclusions}

In conclusion, the 5-year RFS rate in patients who underwent curative resection for gastric GISTs was excellent. Recurrence occurred in $6.2 \%$ of patients with gastric GISTs and was associated with tumor size, mitotic count, and histologic subtype. For risk categorization, the 7th UICC/AJCC TNM staging system may be more useful than the NIH consensus criteria. Further large-scale, multi-center studies with longer follow-up periods are needed to validate our results.

\section{Abbreviations}

AIC: Akaike's Information Criterion; Cl: confidence interval; GISTs: gastrointestinal stromal tumors; HPF: high-power fields; HR: hazard ratio; NIH: National Institutes of Health; RFS: recurrence-free survival; SBC: Schwarz Bayesian Criterion; TNM: tumor-node-metastasis; UICC/ AJCC: Union for International Cancer Control/American Joint Committee on Cancer

\section{Acknowledgements}

We acknowledge assistance with statistical analysis from Pusan National University Hospital Clinical Trial Center Biostatistics Office.

\section{Funding}

GHK and DYP received a grant from the National R\&D Program for Cancer Control, Ministry for Health, Welfare and Family Affairs, Republic of Korea (0920050), and GHK received a grant from the Medical Research Center Program through the National Research Foundation of Korea grant funded by the Korea government (NRF-2015R1A5A2009656).

Availability of data and materials

Data will not be made available in order to protect the participant identity.

\section{Authors' contributions}

CHP reviewed the literature and drafted the manuscript; GHK designed and supervised research; BEL reviewed draft and performed research; GAS, DYP, DHK and TYJ advised and performed research; CHP and GHK analyzed data. All authors read and approved the final manuscript.

\section{Ethics approval and consent to participate}

The study was conducted in accordance with the Declaration of Helsinki and was approved by the Institutional Review Board of Pusan National University Hospital (E-2015216).

\section{Consent for publication \\ Not applicable.}

\section{Competing interests}

The authors declare that they have no competing interests.

\section{Publisher's Note}

Springer Nature remains neutral with regard to jurisdictional claims in published maps and institutional affiliations.

\section{Author details}

'Department of Internal Medicine, Pusan National University School of Medicine, and Biomedical Research Institute Pusan National University Hospital, 179, Gudeok-ro, Seo-Gu, Busan 49241, South Korea. ${ }^{2}$ Department of Pathology, Pusan National University School of Medicine, Busan, South Korea. ${ }^{3}$ Department of Surgery, Pusan National University School of Medicine, Busan, South Korea.

Received: 19 August 2016 Accepted: 24 November 2017 Published online: 06 December 2017

\section{References}

1. Hirota S, Isozaki K, Moriyama Y, Hashimoto K, Nishida T, Ishiguro S, et al. Gain-of-function mutations of c-kit in human gastrointestinal stromal tumors. Science. 1998;279:577-80.

2. Fletcher CD, Berman JJ, Corless C, Gorstein F, Lasota J, Longley BJ, et al. Diagnosis of gastrointestinal stromal tumors: a consensus approach. Hum Pathol. 2002:33:459-65.

3. Heinrich $M C$, Corless $C L$, Demetri $G D$, Blanke $C D$, von Mehren $M$, Joensuu $H$, et al. Kinase mutations and imatinib response in patients with metastatic gastrointestinal stromal tumor. J Clin Oncol. 2003;21:4342-9.

4. Miettinen M, Lasota J. Gastrointestinal stromal tumors: pathology and prognosis at different sites. Semin Diagn Pathol. 2006;23:70-83.

5. Sepe PS, Brugge WRA. Guide for the diagnosis and management of gastrointestinal stromal cell tumors. Nat Rev Gastroenterol Hepatol. 2009:6:363-71.

6. Casali PG, Le Cesne A, Poveda Velasco A, Kotasek D, Rutkowski P, Hohenberger $P$, et al. Time to definitive failure to the first tyrosine Kinase inhibitor in localized GI Stromal tumors treated with Imatinib as an adjuvant: a European Organisation for Research and Treatment of Cancer soft tissue and bone sarcoma group intergroup randomized trial in collaboration with the Australasian gastro-intestinal trials group, UNICANCER, French sarcoma group, Italian sarcoma group, and Spanish Group for Research on sarcomas. J Clin Oncol. 2015;33:4276-83.

7. Dematteo RP, Ballman KV, Antonescu CR, Maki RG, Pisters PW, Demetri GD, et al. Adjuvant imatinib mesylate after resection of localised, primary gastrointestinal stromal tumour: a randomised, double-blind, placebocontrolled trial. Lancet. 2009;373:1097-104.

8. Joensuu H, Eriksson M, Sundby Hall K, Hartmann JT, Pink D, Schutte J, et al. One vs three years of adjuvant imatinib for operable gastrointestinal stromal tumor: a randomized trial. JAMA. 2012;307:1265-72.

9. Agaimy A. Gastrointestinal stromal tumors (GIST) from risk stratification systems to the new TNM proposal: more questions than answers? A review emphasizing the need for a standardized GIST reporting. Int I Clin Exp Pathol. 2010;3:461-71.

10. Dematteo RP, Gold JS, Saran L, Gonen M, Liau KH, Maki RG, et al. Tumor mitotic rate, size, and location independently predict recurrence after resection of primary gastrointestinal stromal tumor (GIST). Cancer 2008;112:608-615.

11. Hassan I, You YN, Shyyan R, Dozois EJ, Smyrk TC, Okuno SH, et al. Surgically managed gastrointestinal stromal tumors: a comparative and prognostic analysis. Ann Surg Oncol. 2008;15:52-9.

12. Rutkowski P, Wozniak A, Debiec-Rychter M, Kakol M, Dziewirski W, Zdzienicki $M$, et al. Clinical utility of the new American joint committee on cancer staging system for gastrointestinal stromal tumors: current overall survival after primary tumor resection. Cancer. 2011;117:4916-24. 
13. Kim MC, Yook JH, Yang HK, Lee HJ, Sohn TS, Hyung WJ, et al. Long-term surgical outcome of 1057 gastric GISTs according to 7th UICC/AJCC TNM system: multicenter observational study from Korea and Japan. Medicine (Baltimore). 2015:94:e1526.

14. Miettinen $M$, Sobin LH, Lasota J. Gastrointestinal stromal tumors of the stomach: a clinicopathologic, immunohistochemical, and molecular genetic study of 1765 cases with long-term follow-up. Am J Surg Pathol. 2005:29:52-68.

15. lesalnieks I, Rummele P, Dietmaier W, Jantsch T, Zulke C, Schlitt HJ, et al. Factors associated with disease progression in patients with gastrointestinal stromal tumors in the pre-imatinib era. Am J Clin Pathol. 2005;124:740-8.

16. Demetri $G D$, von Mehren $M$, Blanke $C D$, Van den Abbeele $A D$, Eisenberg $B$, Roberts PJ, et al. Efficacy and safety of imatinib mesylate in advanced gastrointestinal stromal tumors. N Engl J Med. 2002;347:472-80.

17. Kim $I H$, Kim IH, Kwak SG, Kim SW, Chae HD. Gastrointestinal stromal tumors (GISTs) of the stomach: a multicenter, retrospective study of curatively resected gastric GISTs. Ann Surg Treat Res. 2014;87:298-303.

18. Bertin M, Angriman I, Scarpa M, Mencarelli R, Ranzato R, Ruffolo C, et al. Prognosis of gastrointestinal stromal tumors. Hepato-Gastroenterology. 2007;54:124-8

19. Hsu KH, Yang TM, Shan YS, Lin PW. Tumor size is a major determinant of recurrence in patients with resectable gastrointestinal stromal tumor. Am J Surg. 2007;194:148-52.

20. Reith JD, Goldblum JR, Lyles RH, Weiss SW. Extragastrointestinal (soft tissue) stromal tumors: an analysis of 48 cases with emphasis on histologic predictors of outcome. Mod Pathol. 2000;13:577-85.

21. Martin J, Poveda A, Llombart-Bosch A, Ramos R, Lopez-Guerrero JA, Garcia del Muro J, et al. Deletions affecting codons 557-558 of the c-KIT gene indicate a poor prognosis in patients with completely resected gastrointestinal stromal tumors: a study by the Spanish Group for Sarcoma Research (GEIS). J Clin Oncol 2005;23:6190-6198.

22. Singer $\mathrm{S}$, Rubin BP, Lux ML, Chen CJ, Demetri GD, Fletcher $C D$, et al. Prognostic value of KIT mutation type, mitotic activity, and histologic subtype in gastrointestinal stromal tumors. J Clin Oncol. 2002;20:3898-905.

23. Tran T, Davila JA, El-Serag HB. The epidemiology of malignant gastrointestinal stromal tumors: an analysis of 1,458 cases from 1992 to 2000. Am J Gastroenterol. 2005:100:162-8

24. Bertolini V, Chiaravalli AM, Klersy C, Placidi C, Marchet S, Boni L, et al. Gastrointestinal stromal tumors-frequency, malignancy, and new prognostic factors: the experience of a single institution. Pathol Res Pract. 2008:204:219-33.

25. Choi IJ. Gastric cancer screening and diagnosis. Korean I Gastroenterol. 2009;54:67-76.

26. Tashiro A, Sano M, Kinameri K, Fujita K, Takeuchi Y. Comparing mass screening techniques for gastric cancer in Japan. World J Gastroenterol. 2006;12:4873-4.

\section{Submit your next manuscript to BioMed Central and we will help you at every step:}

- We accept pre-submission inquiries

- Our selector tool helps you to find the most relevant journal

- We provide round the clock customer support

- Convenient online submission

- Thorough peer review

- Inclusion in PubMed and all major indexing services

- Maximum visibility for your research

Submit your manuscript at www biomedcentral.com/submit

) Biomed Central 